\title{
Teknokultura
}

ISSNe: $1549-2230$

http://dx.doi.org/10.5209/TEKN.56687

\section{Los Estudios Críticos de Internet: conceptos, debates y retos ${ }^{1}$}

\author{
Laura Favaro ${ }^{2}$
}

Recibido: 9 de julio de 2017 / Aceptado: 8 de agosto de 2017. Open peer reviews

Resumen. Este artículo ofrece una revisión de la literatura en los Estudios de Internet desde su comienzo hasta la actualidad, prestando particular atención a conceptos y debates centrales, además de cuestiones sobre la interactividad del usuario. Un objetivo principal es elaborar una exposición de los Estudios Críticos de Internet, dado su reconocido interés y su sorprendente ausencia general en la literatura científica en español. Asimismo, el artículo pretende resaltar la importancia de incorporar una mirada feminista en el trabajo teórico así como empírico sobre Internet. En primer lugar se examina una etapa inicial caracterizada por un contraste entre los pronósticos distópicos y las celebraciones optimistas acerca del potencial transformador de Internet. El artículo continúa esbozando los principales interrogantes y conclusiones surgidos en la segunda etapa de los Estudios de Internet, con su más serio compromiso hacia la evidencia empírica y su interés especial en documentar el uso efectivo. La siguiente sección se centra en los términos 'convergencia mediática' y 'cultura participativa'. Por último, se introducen una serie de conceptos clave — donde destaca especialmente el de 'trabajo digital' - para un área de estudio con un abordaje alternativo sobre la interactividad en los contextos online e Internet en general. También se presenta brevemente un caso de estudio como muestra de tal enfoque, así como de la necesidad de una mayor integración del análisis de género en los Estudios Críticos de Internet.

Palabras clave: convergencia mediática; cultura participativa; economía online; estudios feministas de medios de comunicación; trabajo digital.

\section{[en] Critical Internet Studies: concepts, debates and challenges}

\begin{abstract}
This article offers a review of the literature in Internet Studies from its beginning to the present, paying particular attention to central concepts and debates, in addition to issues about user interactivity. One principal objective is to elaborate an exposition of Critical Internet Studies, given its established interest and its surprising general absence in the scholarly literature in Spanish. In addition, the article aims to highlight the importance of incorporating a feminist lens as part of both theoretical and empirical work on the Internet. First it examines an initial stage characterised by a contrast between dystopian predictions and optimistic celebrations about the transformative potential of the Internet. The article continues by outlining the main questions and conclusions that emerged in the second stage of Internet Studies, with its more serious commitment to empirical evidence and documenting actual use. The next section focuses upon the terms 'media convergence' and 'participatory culture'. Finally, I introduce a number of concepts that are key for a field of study with an alternative approach to interactivity in online contexts and the Internet more generally, especially significant being that of 'digital labour'. I also briefly report on a case study as an illustration of such an approach, as well as the need for a greater integration of gender analysis within Critical Internet Studies.
\end{abstract}

1 Este trabajo se inscribe dentro de las líneas de investigación del siguiente proyecto perteneciente al programa de Proyectos EXCELENCIA del Plan Nacional de Investigación: La resignificacion de la mujer-víctima en redes sociales: implicaciones para la construcción de la vulnerabilidad y la resistencia en el activismo on-line (FEM2015-65834-C2-1-P).

2 Universidad Rey Juan Carlos (España)

E-mail: laura.favarog@gmail.com 
Keywords: digital labour; feminist media studies; media convergence; online economy; participatory culture.

Sumario: 1. Introducción. 2. Debates iniciales: dicotomías e hipérboles. 3. La comercialización y el giro empírico. 4. Convergencia mediática y cultura participativa. 5. La economía online y el trabajo digital. 6. Conclusión. 7. Referencias.

Cómo citar: Favaro, L. “Los Estudios Críticos de Internet: conceptos, debates y retos”: Teknokultura, vol. 15 (1) 2018, pp. 151-167.

\section{Introducción}

Los Estudios de Internet son un campo relativamente joven pero ya bien establecido que incorpora disciplinas tan dispares como las Artes, la Psicología y la Informática, siendo la Sociología y los Estudios Culturales y de los Medios especialmente cruciales. Su rápida expansión ha generado una creciente diversidad además de especialización tanto en el ámbito teórico como metodológico, siendo en este último sentido particularmente significativa la ética en la investigación (p.ej. Buchanan, 2011). Este artículo ofrece una revisión de este multidisciplinar y dinámico campo desde su comienzo en los años noventa hasta el momento actual. Presta especial atención a algunos de los principales conceptos y debates, enfatizando cuestiones sobre el uso y en particular la interactividad del usuario, al ser una fuente considerable de desacuerdo y dada su trascendencia, particularmente tras la Web 2.0. Un objetivo principal es elaborar una exposición de los Estudios Críticos de Internet, ya que su interés ha recibido gran reconocimiento académico, sobre todo para abordar temas tales como las relaciones de explotación y la producción de valor en Internet, pero también debido a su sorprendente ausencia general en la literatura científica en español. En un contexto todavía innegablemente masculinizado el artículo también pretende resaltar la importancia para los investigadores críticos de integrar consideraciones y perspectivas feministas en nuestro trabajo teórico así como empírico sobre Internet.

La siguiente sección del artículo delinea la primera etapa de los Estudios de Internet, que comienza alrededor del nacimiento de la web sobre el año 1990, y está caracterizada por el contraste entre los pronósticos distópicos y las celebraciones optimistas acerca del potencial transformador de Internet. Se continúa con una revisión de los principales interrogantes y conclusiones surgidos en la segunda etapa, que emerge un poco antes del estallido de la burbuja de las 'puntocom' a finales de siglo con un más serio compromiso hacia la evidencia empírica y con un interés especial en documentar el uso efectivo de Internet (Livingstone, 2005). Encontrándonos ya a mediados de la década del 2000, cuando la Web 2.0 se adentra en la conciencia colectiva, la siguiente sección examina los términos 'convergencia mediática' y 'cultura participativa'. Seguidamente se presenta un área de estudio con una manera alternativa de abordar la interactividad en los contextos online, e Internet en general. Introduzco una serie de conceptos clave - donde destaca en particular el de 'trabajo digital' - y finalizo con un caso de estudio como muestra de este enfoque, así como de la necesidad de un mayor compromiso con cuestiones de género por parte de los Estudios Críticos de Internet. 


\section{Debates iniciales: dicotomías e hipérboles}

En los primeros años los teóricos de la 'cibercultura' estaban particularmente interesados en explorar las implicaciones socioculturales de las agregaciones formadas a través de lo que a menudo se llamaba comunicación mediada por ordenador. Se dedicó una intensa atención a lo apropiado del término comunidad en este contexto, y a considerar si las entonces denominadas comunidades virtuales constituían comunidades 'auténticas', 'reales' o 'legítimas' (Bordieu y Coleman, 1991). Otro debate notable se centró en sus implicaciones políticas: si tales comunidades eran la panacea tecnológica para muchos de los problemas sociales del mundo contemporáneo (Bromberg, 1996); o si, por otro lado, la experiencia online continuada agravaría la degradación de las comunidades en la 'vida real' (Brook y Boal, 1995), llevando a un mayor aislamiento, anomia y comportamiento asocial en las sociedades postmodernas (Wilson y Peterson, 2002). Para algunos las comunidades virtuales eran un "nuevo mito gobernante" (Lockard, 1997, p. 229) que alentaba el alejamiento del compromiso cívico en el ámbito offline y profundizaba la privatización de la vida social (Doheny-Farina, 1996). Por el contrario, otros las consideraron una herramienta para propiciar la igualdad en las sociedades divididas en clases y saturadas de información, aportando al ciudadano medio enormes ventajas tanto comerciales como de índole intelectual, social "y lo más importante, ventaja política" (Rheingold, 1993, p. 4). A medida que Internet se popularizó a partir de mediados de la década de los noventa, las narrativas sobre la radical potencialidad emancipatoria y democrática del 'ciberespacio' comenzaron a circular aún más ampliamente. Por ejemplo, Lévy (1997) desarrolló la noción de 'inteligencia colectiva', mientras que Pearce (1997) celebraba una 'revolución interactiva', basada en "usar herramientas poderosas para crear nuestras propias experiencias educativas y de entretenimiento en lugar de aceptar pasivamente lo que nos proporcionan los llamados expertos". Más aún, según Pearce (1997, p. xvii): "El código binario es el Esperanto digital que está conduciendo simultáneamente al empoderamiento individual y a la unidad mundial".

En los años noventa Internet también surgió como un área de gran interés además de perspectivas encontradas dentro del ámbito feminista. Por un lado se argumentó que implicaba inevitablemente códigos y valores masculinos. Desde esta perspectiva se señaló, por ejemplo, la estrecha y duradera asociación entre tecnología y masculinidad (Wajcman, 1991), las raíces de Internet en el complejo militar-industrialacadémico estadounidense, junto con el dominio de los varones en la investigación, desarrollo y uso de las TIC, o el androcentrismo de la mayoría de los contenidos online (Van Zoonen, 1992). Asimismo, se recalcó que Internet constituía un entorno hostil para las mujeres. Muchas de las primeras investigaciones feministas demostraron que las usuarias a menudo tenían que lidiar con sexismo y acosos (sobre todo de índole sexual), junto con una dominación discusiva (Kramarae y Taylor, 1993) y resistencia masculina a su participación (Herring et al., 1995). La 'red informática', pues, estaba atravesada por las mismas divisiones de género, desigualdades y relaciones de poder presentes en los otros ámbitos de las sociedades capitalistas occidentales, lo que incluía cuestiones de acceso (Balka, 1993).

En cambio, otra perspectiva feminista consideró Internet como fuente fundamental de nuevas y radicales potencialidades, por ejemplo educativas (Spender, 1995) y de concienciamiento político en nuevos ambientes transnacionales (Youngs, 1999). En ocasiones incluso se llegó a apreciar como singularmente adecuado para las mu- 
jeres y hasta intrínsecamente cercano a ellas. En este sentido destaca la manera en la que Plant (1996) (re)interpretó el desarrollo de redes de información descentralizadas e informales como señal de un proceso de feminización. Plant (1997, p. 144) valoró Internet argumentando que "las conexiones indirectas, sinuosas con las que las mujeres siempre han estado asociadas y la interconexión informal en lo que han sobresalido ahora pasan a ser protocolos para todos". Es más, desde su punto de vista, los "sistemas complejos y mundos virtuales [...] socavan tanto la visión del mundo como la realidad material de dos mil años de control patriarcal" (Plant, 1996, p. 170). Particularmente celebradas por estos relatos feministas más optimistas sobre Internet fueron las comunicaciones anónimas y basadas en texto. Se consideró que estas formas de comunicarse ofrecían potenciales liberadores para trascender las limitaciones de las construcciones patriarcales de género, así como para explorar identidades alternativas y/o múltiples, desinhibidas de la amenaza de sanción social dentro del entorno físico (Reid, 1995). Inspirada por el post-estructuralismo, combinando la política transgénero, la tecnofilia (Van Zoonen, 2002) y basándose en la teoría cyborg de Haraway (1991), una rama central del ciberfeminismo destacó por su acercamiento a las redes como "espacios de transformación, fábricas de identidad" (Stone, 1995, p. 181), donde las mujeres podían finalmente "flotar libres de determinaciones biológicas y socioculturales" (Dery, 1994, p. 3). Pocos años después, como veremos a continuación, elementos de esta segunda perspectiva fueron extensamente cuestionados.

\section{La comercialización y el giro empírico}

A partir del final de los noventa surge la segunda etapa de los Estudios de Internet, marcada por un interés en su documentación sistemática e investigaciones fundamentadas empíricamente, en particular respecto a sus proliferantes usuarios y usos (Wellman, 2011). Algunos hechos determinantes incluyen el lanzamiento de revistas científicas como Information, Communication and Society (1998), la formación en 1999 de la Association of Internet Researchers, y la celebración de su primer congreso un año después. Durante este periodo se puso de relieve la manera en la que los primeros debates estuvieron a menudo basados en conjeturas y evidencias anecdóticas (Wellman, 2011), o centrados en espacios no comerciales y agregaciones sociales ad hoc, tales como grupos de noticias (Watson, 1997), comunidades de fans (Baym, 1995), salas de chat basadas en identidades sexuales (Correll, 1995) y los juegos de rol mazmorra/dominios multiusuario (Turkle, 1995). En consecuencia, se reclamó una ampliación del campo de estudio a "las prácticas más mainstream y cotidianas de uso", tarea que fue percibida como "especialmente relevante a medida que Internet y la World Wide Web se vuelven más ubicuas y más comercializadas" (Consalvo y Paasonen, 2002, p. 4).

Las posibilidades que se celebraron en un momento inicial fueron pronto atenuadas en gran medida por la irrupción de la construcción de Internet como una herramienta para el comercio y la mercantilización de las audiencias, y, de manera interrelacionada, debido al aumento de la propiedad por parte de los grandes conglomerados mediáticos (Worthington, 2005). Un objetivo principal de las empresas fue comercializar productos y servicios a determinados grupos demográficos, con el fin de competir con los portales universales establecidos como Yahoo, AOL o MSN 
y para sacar provecho de los usuarios emergentes, creando contenidos cada más segregados y orientados a nichos (Royal, 2005). En el centro de este giro separatista impulsado por el comercio, que comenzó al final de los noventa para continuar intensificándose, está la profusión de productos y servicios diseñados para mujeres. El modelo por excelencia que nace de este proceso se basa en ofrecer contenido parecido a las revistas femeninas, además de posibilidades de compra y una nueva característica estrella: los foros de discusión, haciendo hincapié en la idea de una comunidad femenina (Favaro, 2017). Ciertamente, la comercialización de la web llevó a centrar el "consumismo y la duplicación de formas familiares de medios populares de masas" (Seiter, 2003, p. 689), junto con las comunidades de usuarios, con el fin de cultivar su lealtad e inversión emocional y aumentar la adherencia (stickiness) de los sitios (Jarrett, 2003).

Es precisamente en este contexto cuando Paasonen (2002, p. 89) argumentó que "Los servicios comerciales para las mujeres que llegan a un vasto público de usuarias de la red están muy lejos de las fantasías académicas de géneros fluidos”. Un número creciente de estudios mostraron cómo los cambios asociados con el mainstreaming y la comercialización ocasionaron formas de organización y uso que se alejaron rápidamente de las visiones de 'taller de identidad' o 'laboratorio de género'; por ejemplo apuntando a la preocupación por que los cuerpos estén 'auténticamente' sexuados y generizados (Consalvo y Paasonen, 2002; Sundén, 2002) —una preocupación tanto ideológica como producto de imperativos de marketing. También dirigiéndose a las afirmaciones optimistas iniciales, Liesbet van Zoonen alegaba en 2002: "Hay suficiente evidencia sobre pornografía (infantil), extremismo derechista, acoso sexual, entre otros elementos desagradables para rechazar cualquier visión utópica de Internet como un ambiente femenino no problemático" (Van Zoonen, 2002, p. 11). Van Zoonen (2002) remarcó además cómo algunas teóricas feministas estaban, aunque inintencionadamente, alineándose con los expertos de marketing - $-\mathrm{y}$ su esencialismo de género - que en ese momento comenzaban a también caracterizar con entusiasmo la tecnología como particularmente cercana y empoderante para las mujeres.

En conjunto, a diferencia de las primeras evaluaciones sobre las enormes transformaciones que traería Internet, pronto se hizo evidente que "los cambios han sido menos dramáticos y más enraizados en las prácticas existentes y relaciones de poder de la vida cotidiana" (Wilson y Peterson, 2002, p. 449). Se observó que ni las esperanzas utópicas sobre la comunicación y democracia global ni los temores distópicos sobre el final del contacto interpersonal tuvieron lugar (Wellman, 2011) — la realidad era mucho más matizada y compleja. La rígida división o estricta dualidad entre lo 'viejo' y lo 'nuevo' y entre lo 'real' y lo 'virtual' se problematizó y complicó, y cada vez más la actividad online fue abordada como parte de, y tanto configurada por y configurando, el mayor espectro de la experiencia vivida (Kendall, 1999). Especialmente a medida que la integración de Internet en la vida cotidiana aumentaba, se rechazaron los discursos escapistas y las concepciones sobre los nuevos medios como constituyentes de mundos separados (Haythornthwaite y Wellman, 2002). Al contrario, se reivindicó un modelo de investigación que contextualiza "la actividad mediática como parte de un más amplio terreno social de experiencia" (Gray, 2009, p. 86), y que busca entender mejor el entretejido entre ambos (Baym, 2006). Ilustrando este importante giro conceptual fue el cambio terminológico de 'virtual/real' a 'on/off-line', y de las discusiones acerca del 'ciberespacio' a hablar sobre 'Internet' (Hardey, 2002). Del mismo modo, la metodología etnográfica cobró particular 
significancia (Hine, 2000). En contraste con gran parte de las tendencias iniciales, los estudios feministas comenzaron a evitar tanto el esencialismo de género como el determinismo tecnológico, concibiendo la relación entre género y tecnología como situada, fluida y mutuamente constituyente (Wajcman, 2010).

La incorporación de Internet como parte integral de la vida cotidiana de las masas y el florecimiento de la Web 2.0 han fomentado por un lado la consolidación de los Estudios de Internet, reuniendo a académicos de diversas áreas en las Ciencias Sociales, Humanidades e Informática, y por el otro la integración de la investigación sobre Internet dentro de los congresos y revistas mayoritarias de un constantemente creciente número de disciplinas. Estas son las dos distintas pero complementarias tendencias que para Barry Wellman (2011) definen a la tercera etapa de los Estudios de Internet, la que denomina: 'de la documentación al análisis'. Algunos de los conceptos y debates clave de tal etapa —o de la era de la Web Social— se consideran en las siguientes secciones.

\section{Convergencia mediática y cultura participativa}

Tanto en los ámbitos académicos y de políticas de medios así como en la industria y la literatura popular, el concepto de convergencia ha demostrado ser de gran utilidad para abordar un amplio número de aspectos sobre la comunicación y los medios contemporáneos en general y como identificador de múltiples tendencias en la cultura mediática digital en particular (Grant y Wilkinson, 2009). La convergencia, afirma Burgess (2017, p. 47), es "una de las dinámicas constitutivas de los nuevos medios", caracterizados por el derrumbe de distinciones, fronteras difusas o procesos de hibridación. La mayoría de los usos del término apuntan a la fusión de industrias, componentes y plataformas, y por lo tanto también al aumento de contenido cross-media ('convergencia tecnológica'). Es decir, la convergencia implica el paso de los contenidos específicos a un medio hacia aquellos que fluyen por múltiples canales. También se han destacado los procesos de convergencia con respecto a la creación de contenido y servicios de marketing, a la creatividad a nivel individual y la producción comercial masiva (Deuze, 2008), junto con la intersección de medios grassroots y los corporativos (Jenkins, 2006). Asimismo, la creciente convergencia de la propiedad, la regulación y la producción ha sido enfatizada por comentaristas críticos (p.ej. Fuchs, 2011) que, a la luz de crecientes adquisiciones y fusiones, han expresado su preocupación por una industria de (cross/trans-) media cada vez más concentrada y jerarquizada bajo el control de un número progresivamente reducido de gigantes conglomerados transnacionales ('convergencia corporativa'). La creciente liquidez entre seres humanos y máquinas así como los dominios público y privado ha sido igualmente fruto de atención, junto a la convergencia entre "el yo e identidades sociales (especialmente en las redes sociales), entre trabajo y juego, y debido a la compresión tiempo-espacial, la convergencia de lo local y lo global" (Deuze, Blank y Speers, 2012, p. 11).

Por lo tanto, el concepto de convergencia mediática circunscribe simultáneamente procesos tecnológicos, industriales y socioculturales, y está íntimamente relacionado con los de globalización y transnacionalismo. Describe una época prolongada de transición y transformación en la operación de los medios de comunicación (Jenkins, 2006), e inevitablemente plantea interrogantes sobre cómo estudiar la ecología me- 
diática contemporánea - poniendo especialmente en cuestión la división tradicional entre el estudio de la industria y el de las audiencias (Jenkins y Deuze, 2008), ya que, y sobre todo con Internet, el consumo o uso de medios incluye crecientemente algún tipo de producción. Esta 'convergencia cultural' se ve reflejada en un nuevo lenguaje específico, como los acrónimos 'prosumidor' (Toffler, 1980) y el más reciente 'produso' (Bruns, 2007). También estrechamente asociado al de convergencia, el término 'cultura participativa' designa un escenario mediático que prioriza una audiencia activa y que interpela cada vez más a los consumidores como una fuerza productiva en la creación y distribución de contenido (Jenkins, 2006).

Las narrativas entusiastas sobre la participación, la construcción de comunidades y el empoderamiento de los usuarios se han incrementado con el auge de la Web 2.0. Éstas permean la industria de los medios, pero también sectores del mundo académico. Por ejemplo, para Axel Bruns (2007, p. 100) el "software social o entornos Web 2.0" manifiestan un cambio de paradigma hacia "una nueva era de la información dirigida por el usuario", pronosticando un modelo democrático basado en las actividades de los 'produsarios'. De manera similar Jenkins (2006, p. 9) sostiene que la convergencia mediática ha ocasionado una cultura participativa constituida por consumidores "recientemente empoderados". Esta cultura, argumenta Jenkins (2006, p. 4), conduce a una inteligencia colectiva, entendida "como una fuente alternativa de poder mediático". Jenkins (2006) reconoce algunas de las problemáticas asociadas con la convergencia, por ejemplo la propiedad concentrada de los medios. Asimismo, este autor señala junto con Deuze que "el contenido generado por el usuario existe tanto dentro como fuera de los contextos comerciales, y apoya así como subvierte el control corporativo" (Jenkins y Deuze, 2008, p. 7). Según Jenkins y Deuze (2008, p. 7), "el paradigma de la Cultura de la Convergencia representa un intento de captar este contradictorio panorama mediático". Sin embargo, tal paradigma se ha asociado con posiciones demasiado optimistas, incluso simplistas, y ha sido objeto de una serie de fuertes críticas (Hay y Couldry, 2011).

Para Christian Fuchs (2009, p. 96) los enfoques de la cultura participativa demuestran un "nuevo optimismo tecno-determinista" semejante a la "ideología que acompañó el ascenso comercial de Internet en los años noventa" y constituyente de una "ideología web 2.0". Con referencia al libro Convergence Culture (Jenkins, 2006) específicamente, Driscoll y Gregg (2011, p. 567) declaran que "el análisis ideológico $[\ldots]$ es sumamente necesario cuando los estudios culturales, de los medios y especialmente de Internet parecen preparados para servir como los profetas para nuevas industrias". Recalcando el énfasis de Jenkins sobre el fandom, Driscoll y Gregg (2011, p. 567) además advierten a los investigadores de Internet contra nuevamente "permitir que las prácticas de una minoría representen la visión optimista del inminente panorama mediático". También cuestionando argumentos de Jenkins (2006), Andrejevic (2011a, p. 613) enfatiza que las empresas emplean narrativas sobre comunidad y participación como "estrategias para gestionar audiencias interactivas", donde "El objetivo no es tanto negociar colectivamente con grupos de interés como un seguimiento encubierto y preventivo de opiniones a una escala sin precedentes". Por su parte, Deuze (2008) destaca cómo gran parte de la cultura mediática contemporánea está fuertemente regulada, restringida e incrustada dentro de procesos empresariales que pretenden no liberar sino sacar provecho de la participación del usuario. 
Otras críticas emanan del análisis feminista. Aquí teóricas han destacado la manera en la que los estudios de la convergencia mediática y la cultura participativa han tendido a dejar de lado cuestiones de género. Al igual que a los hombres, la Web 2.0 ha proporcionado a las mujeres oportunidades sin precedentes para relacionarse y comunicar, para acceder, producir y distribuir medios e información, como fuente de placer, creatividad y colaboración. Asimismo, Internet se ha convertido en un espacio fundamental para desarrollar, difundir y vincularse con ideas feministas, para establecer contactos y organizarse a nivel tanto nacional como global, aumentar la concienciación y reclutar voluntarios, además de producir nuevas identidades y prácticas $^{3}$. Todo esto coexiste, sin embargo, con un alto nivel de hostigamiento claramente enraizado en el sistema de género. Enfrentarse en entornos online a contactos no deseados, comentarios sexistas, insultos, intimidaciones e incluso amenazas de violación y muerte se ha convertido en un rasgo común para las mujeres activas en la vida pública y/o comprometidas abiertamente con el feminismo (Jane, 2014)4. En parte como respuesta a una reciente mayor visibilidad del feminismo popular, esta misoginia en red o interconectada (networked misogyny) (Banet-Weiser y Miltner, 2016) está cada vez más dirigida hacia todas las usuarias. En otras palabras, los usos feministas de Internet coexisten con una fuerte resistencia misógina, al igual que una web altamente comercializada y profundamente cimentada en posicionar a las mujeres como sujetos a la vez que objetos de consumo (Favaro, 2017). Adicionalmente, a raíz del surgimiento de la 'autenticidad' como elemento normativo de la interacción online, hay una creciente expectativa por que el usuario reproduzca su identidad 'real' y presente tanta información personal como sea posible. En el centro de esta querencia por lo 'auténtico' están las clasificaciones de género, hasta el punto en el que "el género, primordialmente en combinación con la heteronormatividad, puede considerarse la categoría más importante en la autoconstrucción de la identidades de los usuarios" (Carstensen, 2009, p. 113). Más aún, la transformación de Internet en una experiencia visual ha desplegado una intensa fascinación por la exposición fotográfica de la existencia físicamente situada, y con ella esquemas de clasificación offline, especialmente el sistema sexo/género (Van Doorn, Wyatt y Van Zoonen, 2008).

Cuestionando las posibilidades liberadoras de la interactividad que celebran algunos estudiosos de los nuevos medios como Jenkins, Ouellette y Wilson (2011, p. 549) resaltan "la división sexual del trabajo como una dimensión desatendida de la cultura de la convergencia y del ethos participativo que se asume que engendra". Estas autoras observan cómo las tecnologías y plataformas convergentes promueven la expectativa de que las mujeres hagan un uso emprendedor de los medios para el auto-trabajo activo y la gestión familiar exitosa. Así, argumentan que la participación en el ecosistema mediático contemporáneo implica para las mujeres un 'segundo turno' de trabajo afectivo y doméstico/familiar, situado dentro de un régimen político de privatización y responsabilidad personal más amplio. Bajo la cultura de la convergencia, impulsada por las nuevas plataformas de medios, la interactividad de las mujeres se moviliza como una exigencia de ciudadanía neoliberal generiza-

\footnotetext{
Para más información, se puede revisar el trabajo realizado por Keller (2015), a modo de ejemplo.

4 Esta agresión machista ha llegado a tal punto que ejerce como fuerza silenciadora o disuasoria. Por ejemplo, el recibir frecuentemente trolling misógino, discursos de odio y amenazas de violencia está llevando a algunas mujeres a cuestionarse y hasta cesar su labor como periodistas (Favaro, 2017).
} 
da (Ouellette y Wilson, 2011). De manera similar, Driscoll y Gregg (2011, p. 567) consideran que el modelo de convergencia no registra los imperativos estructurantes de género así como las múltiples y a menudo simultáneas formas de ocio y trabajo en las plataformas digitales; mientras Deuze (2008, p. 31) enfatiza cómo "la apropiación corporativa de los medios [...] abre nuevas oportunidades para las empresas para alistar el 'trabajo gratuito' (Terranova 2000) de los usuarios”. Especialmente con el surgimiento de las redes sociales, el debate sobre el trabajo digital del usuario ha incrementado su relevancia para los estudios críticos acerca de Internet, una importante corriente que considero a continuación (Scholz, 2013).

\section{La economía online y el trabajo digital}

Partiendo de la sospecha respecto a las promesas de los nuevos medios y su retórica de empoderamiento emerge una rama de estudio que ha sustituido el énfasis sobre la cultura participativa por una exploración más profunda de la nueva economía online, analizando en especial la productividad en los contextos digitales a través de los marcos teóricos del trabajo inmaterial (Hardt y Negri, 2004) y gratuito (Terranova, 2000), basados en principios del marxismo autonomista italiano. Esta considera que Internet depende de la extracción de valor de cantidades enormes y continuas de trabajo, gran parte del cual es un trabajo no pagado o gratuito (free labour). Se plantea que la industria corporativa invierte en tecnologías digitales para redefinir la relación entre las esferas de producción y consumo, utilizando la interactividad y participación del usuario de diversas maneras como formas de trabajo que generan productos mercadeables y otros tipos de valor comercialmente explotable. Por ejemplo, centrándose en las comunidades comerciales online, Campbell (2008) se ha basado en el modelo de Jhally y Livant (1986) sobre el 'trabajo de observar' (work of watching) para argumentar que los usuarios realizan un tipo de trabajo beneficioso para las empresas de Internet paralelo al de las audiencias de la televisión comercial, es decir: exponerse a la publicidad a cambio del acceso a recursos culturales/contenidos. Sin embargo, señala Campbell (2008), Internet añade una nueva dimensión a este arraigado contrato implícito entre productores y consumidores de medios, ya que los usuarios también generan contenido. Como Tiziana Terranova (2000, p. 42) observó en su influyente intervención, "las capacidades productivas del trabajo inmaterial en Internet", como "el trabajo de escribir/leer/gestionar y participar en listas de correo/ sitios web/chatlines" amplia significativamente -o incluso constituye - el valor de los espacios. Los usuarios, Terranova (2000, p. 49) subraya, "mantienen un sitio web vivo a través de su trabajo, las horas acumuladas de acceso (generando así publicidad), escribiendo mensajes, participando en conversaciones, y a veces dando el salto a colaboradores". Bajo esta economía online, pues, la 'audiencia como mercancía' (audience commodity) (Smythe, 1977) se convierte en una 'mercancía produsora' (produser commodity) (Fuchs, 2009).

También inspirado por Jhally y Livant, y preocupado por la creciente vigilancia corporativa, Andrejevic afirma que los medios interactivos añaden otra dimensión más a estas nuevas configuraciones. En respuesta a ofertas de conveniencia, personalización y participación, los consumidores de medios digitales comerciales se someten a una vigilancia integral y, de este modo, producen datos explotables para la personalización masiva y el marketing dirigido. Del mismo modo, las empresas emplean las 
comunidades en red como foros para prácticas de auto-revelación donde se desvelan aspectos personales de maneras cada vez más detalladas y exhaustivas. Por consiguiente, para Andrejevic (2004, p. 197), los usuarios "no están tanto participando, en el sentido progresivo de autodeterminación colectiva, como están trabajando al someterse a vigilancia interactiva". Este 'trabajo de ser observado' (work of being watched), Andrejevic (2011b, p. 90) apunta, facilita convertir "detalles de la actividad que antes eludían formas sistemáticas de extracción de valor" en "mercancías informativas". Estas son mercancías cada vez más importantes ya que a medida que Internet amenaza las formas tradicionales de ingreso comercial: "la personalización basada en datos, la predicción y la targetización se convierten en el modelo predeterminado para financiar las infraestructuras de medios comerciales de la era digital" (Andrejevic, 2011a, p. 618).

Así pues, la web depende de los usuarios como proveedores de diversas formas de producción técnica además de "formas de trabajo que no reconocemos inmediatamente como tales" (Terranova, 2000, p. 38). Muchas de estas, afirma Terranova (2000), demuestran un nivel de deseo o placer en el proceso de producción del tipo que se ha teorizado principalmente en relación al consumo en los Estudios Culturales. Como explica Gregg (2009, p. 209), en este ámbito la noción de 'trabajo afectivo' se ha utilizado para referirse a la "actividad humana significativa y productiva que no resulta en un beneficio financiero directo o un valor de cambio, sino que produce un sentido de comunidad, estima, y/o pertenencia para quienes comparten un interés común", como serían los grupos de fans. Especialmente debido a la explosión de contenido generado por el usuario, estas prácticas han recibido una creciente atención en los Estudios Críticos de Internet. En su mayoría las obras destacan por un intento explícito de esclarecer cómo el placer y la explotación están entrelazados en el trabajo digital gratuito que demanda la nueva economía online — véase por ejemplo el artículo de Postigo (2009) sobre el 'trabajo apasionado' de los voluntarios de America Online o el 'trabajo de devoción' hacia las marcas en las comunidades diseñadas por corporaciones que examina Campbell (2008). Como sostiene Terranova (2000, p. 33), el trabajo gratuito online es al mismo tiempo "voluntariamente otorgado y no asalariado, disfrutado y explotado". Esta línea de estudio también acentúa que, aunque esenciales para la economía digital, tipos de trabajo como el cultural y el afectivo - así como el gratuito en general- no se limitan a Internet, sino que son intrínsecos a la infraestructura de las sociedades capitalistas avanzadas, y a su 'factoría social' en particular (Terranova, 2000).

Parece importante también aclarar que investigar las maneras en las que la interactividad se duplica como formas de trabajo no implica descartar las posibilidades de derivar valor afectivo, intelectual o didáctico, o de tomar parte en actividades creativas o en la construcción de relaciones significativas. No niega tampoco que las prácticas de los usuarios tengan potencial para el empoderamiento (Andrejevic, 2004). Del mismo modo, argumentar que "la promesa de la revolución digital se convierte en una coartada para las depredaciones de formas cada vez más agresivas de globalización neoliberal" no significa minimizar cómo los medios digitales se emplean también para desarrollar plataformas de comunicación independientes y alternativas al igual que nuevas formas de activismo social y organización política de resistencia (Andrejevic, 2011c, p. 2). Como explica Andrejevic (2009, p. 36), los Estudios Críticos de Internet lo que proponen es desafiar "la ideología de medios 2.0", basada en una "asociación automática entre la participación interactiva y el 
empoderamiento democrático". Pone en duda el vínculo directo entre medios que promueven la interacción con lo políticamente progresivo, incluso subversivo, siendo esta una idea simplificadora que tanto sirve para reforzar las estrategias comerciales de la cultura corporativa digital. Se trata también de resaltar la manera en la que "las tecnologías de los nuevos medios se están utilizando en muchos contextos de acuerdo con las prioridades que reproducen las mismas formas de alienación que prometen superar" (Andrejevic, 2011c, p. 2), por ejemplo en relación con el poder y el control sobre la información. El estudio crítico de Internet tiene además como objetivo destacar la importante línea que separa "el acceso a los medios de producción de contenido online y la posesión o control de estos recursos" (Andrejevic, 2011b, p. 97). Esta es una línea que se hizo evidente de manera contundente, por ejemplo, cuando las usuarias en las webs femeninas comerciales se enfrentaron al cierre permanente de los foros de discusión, como veremos brevemente más abajo.

A pesar de su preocupación por las relaciones de poder, los marcos dominantes sobre el trabajo gratuito e inmaterial (incluido el afectivo) en los medios digitales, así como los Estudios Críticos de Internet en general, han prestado escasa consideración a cuestiones de género (ver también Daniels, 2013, para un argumento similar sobre la 'raza' y el racismo). Teóricas feministas han destacado, igualmente, la larga trayectoria feminista que sitúa el trabajo no remunerado e inmaterial como fundamental para la economía capitalista. En este sentido, Jarrett (2014) comenta: "A menudo parece como si el trabajo inmaterial sólo fue 'inventado' cuando salió de la cocina y hacia Internet" (p. 15); acentuando asimismo la importancia de que las percepciones e investigaciones feministas sobre los tipos de trabajo afectivo e inmaterial históricamente asociados con las mujeres "no se pierda en la novedad atribuida a los medios digitales" (p. 26). Por su parte, Driscoll y Gregg (2011, p. 567) subrayan la necesidad de realizar estudios que indaguen en las maneras en las que "el trabajo remunerado y no remunerado, y sus diversas formas de reconocimiento financiero, continúan estando generizadas". Un ejemplo es la ya mencionada obra de Ouellette y Wilson (2011), que demuestra cómo en contraste con la afirmación de Andrejevic de que la cultura de la convergencia universaliza las formas de explotación asociadas al trabajo no remunerado femenino, existe una persistente división sexual del trabajo que demanda de las mujeres un segundo turno o jornada laboral no remunerada basada en el trabajo doméstico y afectivo.

Otro ejemplo de incorporación del análisis de género en los Estudios Críticos de Internet es la extensa investigación que hemos realizado sobre las webs o revistas online femeninas (Favaro, 2017). Estos productos mediáticos comerciales están proliferando y progresivamente desbancando a las publicaciones en papel. Su éxito reside en ofrecer contenido similar gratuitamente y "al alcance de un click" (como celebra la popular publicación Cosmopolitan España), así como mayores oportunidades para interactuar, a menudo a través de foros de discusión. Sin embargo, estos espacios interactivos están actualmente desapareciendo, siendo sustituidos por un énfasis cada vez mayor en las redes sociales. Hemos analizado los múltiples determinantes de este nuevo modelo de interacción, en base a 68 entrevistas con profesionales de la industria en España y en el Reino Unido, contenido generado por las usuarias, y una variedad de prensa especializada. El análisis demuestra que el cambio de los foros a las redes sociales - actualmente la fuente principal de tráfico para estas webs - en parte responde a un esfuerzo corporativo por extraer nuevas modalidades de trabajo digital gratuito de las usuarias. Esto incluye de manera notable la intensificación del 
'trabajo de ser observado' (Andrejevic, 2004), ya que las redes sociales facilitan un nivel de vigilancia comercial sin precedentes, intensificado todavía más por los teléfonos móviles, cada vez más el dispositivo de acceso principal. También crucial para estas empresas mediáticas es el trabajo de diseminación de contenido constante al público destinatario, en tanto en cuanto que las jóvenes comparten contenido a través de las redes sociales — convirtiéndose en el nuevo sujeto-objeto ideal de la economía online, el que ya más que un 'produsuario' es un compartidor compulsivo (shareaholic). Este es un cambio paradigmático que forma parte del nuevo modelo de negocio que Jenkins et al. (2013) llaman 'propagabilidad' (spreadability) ${ }^{5}$ —reemplazando al de 'pegajosidad' - y donde "las marcas entran en los espacios donde la gente ya vive e interactúa", beneficiándose de la lógica de la 'economía del regalo' (gift economy) (Green y Jenkins, 2011, p. 117). Esta nueva fase de evolución en la ecología mediática se puede observar claramente en el discurso de los kits de medios de las webs/revistas femeninas, donde se promete a los anunciantes una consumidora que es una "compartidora social activa"; lo que es más: "compartir su vida online es parte de su ritual diario" (The Debrief, 2016). Los estrategas del comercio en Internet también celebran que las redes sociales "aumenta[n] la receptividad a la publicidad" (Morgan, 2015). Esto pone de manifiesto cómo, en palabras de Jarrett (2014, p. 24), "la agencia de los usuarios no está en simple oposición a las relaciones explotadoras del capitalismo sino que está profundamente implicada en su mantenimiento".

Hemos demostrado que el paso del 'produso' a la propagación en las webs femeninas también responde a una doctrina corporativa de control sobre el discurso de la usuaria (García-Favaro, 2016). Tanto estas webs como sus anunciantes, dedicados principalmente a la moda y belleza, consideran que las conversaciones sobre sexo son demasiado explícitas y en última instancia perjudiciales para la 'imagen y reputación' de marca. Al cerrar los foros estas empresas pretenden, además, silenciar las críticas a las líneas editoriales y productos o servicios publicitados. A pesar de todos sus esfuerzos, las webs femeninas no tienen el control absoluto del discurso de las usuarias, ni pueden silenciar la crítica. Al contrario, lidiar con críticas a las decisiones editoriales expresadas a través de las redes sociales es un elemento cotidiano para el equipo de redacción. Sin duda las publicaciones encuentran maneras de beneficiarse de este 'trabajo de resistencia' de las usuarias, por ejemplo simplemente al ganar visibilidad de marca e incluso visitas. También incorporan elementos de la crítica y la desafección — un proceso particularmente característico del 'capitalismo cool' (McGuigan, 2009). Aun así, como dice Jarrett (2014, p. 26):

Los enfoques del marxismo autónomo nos recuerdan cómo la cooperación social de los usuarios y los regímenes afectivos implicados en los medios digitales están siempre en exceso de 'comodificación' y del proceso de trabajo capitalista. Este exceso es potencialmente disruptivo.

No obstante, aunque el ejercicio de control por parte de las webs femeninas tiene diferentes grados de éxito, su nuevo modelo de interacción basado en las redes socia-

5 Ejemplificando lo que consideramos una problemática 'convergencia' entre la industria y la academia, la investigación para la obra Spreadable Media (Jenkins et al., 2013) fue "financiada por los miembros del Convergence Culture Consortium, incluyendo GSDM Advertising, MTV Networks, y Turner Broadcasting” (Jenkins, 2009). 
les problematiza las persistentes celebraciones tanto en la industria como en algunos ámbitos académicos sobre un ecosistema mediático digital cada vez más democrático y liderado por el usuario. Es este panorama complejo, casi siempre contradictorio y a menudo ambivalente, el que los Estudios Críticos de Internet aspiran a esclarecer a través de un análisis concienzudo del poder.

\section{Conclusión}

En contraste con el enfoque instrumental y/o festivo que caracteriza a otros planteamientos académicos - y claramente a los comerciales - los Estudios Críticos de Internet centran cuestiones sobre ideología y explotación, abogando por un Internet cooperativo, libre de dominación y basado en el dominio colectivo público (Fuchs, 2011). Impulsado por el compromiso con la justicia social y la esperanza en la posibilidad de cambio, es un campo que ofrece una creciente riqueza teórica informada por una sólida base en la continua investigación empírica. En general trasciende los marcos binarios con análisis más complejos, como es el caso de las contradictorias dinámicas del poder y la resistencia online, así como más completos, por ejemplo al explorar tanto lo emergente como lo residual, desde los niveles micro a los macro, y combinando elementos de los estudios culturales y la economía política. Al igual que ocurre con los medios 'antiguos', el potencial democrático y emancipador de Internet se ve inevitablemente obstaculizado por las estructuras de poder que forman el contexto sociocultural, político y económico en el que se concibe, desarrolla y usa. Es este un contexto claramente capitalista; pero también, no debemos olvidar, patriarcal. Los Estudios Críticos de Internet demuestran una desacertada atención insuficiente a cuestiones de género: es incuestionable que todo lo relativo a Internet está plenamente generizado. De la misma manera que se ha llegado a considerar como fundamental incorporar aspectos del análisis marxista (Fuchs y Dyer-Witheford, 2013), la integración de una perspectiva feminista es imprescindible para un desarrollo más completo - y en definitiva correcto - del conocimiento crítico sobre Internet.

\section{Referencias}

Andrejevic, M. (2004). The webcam subculture and the digital enclosure. En N. Couldry y A McCarthy, MediaSpace: Place, Scale and Culture in a Media Age (pp. 56-76). London: Routledge.

Andrejevic, M. (2009). Critical media studies 2.0: An interactive upgrade. Interactions: Studies in Communication and Culture, 1(1): 35-51.

Andrejevic, M. (2011a).The Work That Affective Economics Does. Cultural Studies, 25(45): 604-620.

Andrejevic, M. (2011b). Social network exploitation. En Z. Papacharissi, A Networked Self: Identity, Community, and Culture on Social Network Sites (pp. 82-111). New York: Routledge.

Andrejevic, M. (2011c). Estrangement 2.0. World Picture 6. Disponible en: http://www. worldpicturejournal.com/WP_6/Andrejevic.html [2016, 22 de octubre].

Balka, E. (1993). Women's access to on-line discussions about feminism. Electronic Journal of Communication, 3(1). Disponible en: http://www.cios.org/EJCPUBLIC/003/1/00311. HTML [2016, 30 de noviembre]. 
Banet-Weiser, S. y Miltner, K. (2016). \#MasculinitySoFragile: Culture, Structure, and Networked Misogyny. Feminist Media Studies, 16(1): 171-174.

Baym, N. (1995). The emergence of community in computer-mediated communication. En S. Jones, Cybersociety: Computer-mediated Communication and Community (pp. 138163). Thousand Oaks, CA: Sage.

Baym, N. (2006). Finding the quality in qualitative research. En D. Silver y A. Massanari, Critical Cyberculture Studies (pp. 79-87). New York University Press: New York, NY.

Bourdieu, P. y Coleman, J.S. (1991). (Eds) Social Theory for a Changing Society. Boulder, CO: Westview Press.

Bromberg, H. (1996). Are MUDs communities? Identity, belonging and consciousness in virtual worlds. En R. Shields, Cultures of Internet: Virtual Spaces, Real Histories, Living Bodies (pp. 143-152). London: Sage.

Brook, J., \& Boal, I. A. (1995). Resisting the Virtual Life: The Culture and Politics of Information. San Francisco: City Lights.

Bruns, A. (2007). Produsage: Towards a Broader Framework for User-Led Content Creation. Proceedings of the 6th ACM SIGCHI conference on Creativity \& Cognition, 99-106. Disponible http://eprints.qut.edu.au/6623/1/6623.pdf

Buchanan E. A. (2011). Internet research ethics: Past, present, future. En M. Consalvo y C.M. Ess, The Handbook of Internet Studies (pp. 83-108). Oxford: Oxford University Press.

Burgess, J. (2017). Convergence. En L. Ouellette y J. Gray, Keywords for Media Studies (pp. 47-48). New York: New York University Press.

Campbell, J. (2008). Virtually Home: The Commodification of Community in Cyberspace. Tesis doctoral, University of Pennsylvania.

Carstensen, T. (2009). Gender trouble in web 2.0: Gender perspectives on social network sites, wikis and weblogs. International Journal of Gender, Science and Technology, 1(1): 106-127.

Consalvo, M. and Paasonen, S. (2002). On the Internet, women matter. En M. Consalvo y S. Paasonen, Women and Everyday Uses of the Internet: Agency and Identity (1-18). New York: Peter Lang.

Correll, S. (1995). The ethnography of an electronic bar: The lesbian cafe. Journal of Contemporary Ethnography, 24(3), 270-298.

Daniels, J. (2013). Race and Racism in Internet Studies: A Review and Critique, New Media and Society, 15(5): 695-719.

Dery, M. (1994). Flame Wars: The Discourse of Cyberculture. Durham, NC: Duke University Press.

Deuze, M. (2008). Corporate appropriation of participatory culture. En N. Carpentier y B. De Cleen, Participation and Media Production: Critical Reflections on Content Creation (pp. 27-40). Newcastle upon Tyne, UK: Cambridge Scholars Publishers.

Deuze, M., Blank, P. y Speers, L. (2012). A Life Lived in Media. Digital Humanities Quarterly, 6(1).

Doheny-Farina, S. (1996). The Wired Neighborhood. New Haven, CT: Yale University Press.

Driscoll, C. y Gregg, M. (2011). Convergence culture and the legacy of feminist cultural studies. Cultural Studies, 25(4-5): 566-584.

Duffy, B. E. (2013). Remake, Remodel: Women's Magazines in the Digital Age. UrbanaChampaign, IL: University of Illinois Press.

Dutton, W. H. (2013). Internet Studies: The Foundations of a Transformative Field. En W. H. Dutton, The Oxford Handbook of Internet Studies (pp. 1-23). Oxford: Oxford University Press. 
Ess, C. y Consalvo, M. (2011). Introduction: What is 'Internet Studies'? En M. Consalvo y C. Ess, The Handbook of Internet Studies (pp. 1-8). Oxford: Wiley-Blackwell.

Favaro, L. (2017). Transnational Technologies of Gender and Mediated Intimacy. Tesis doctoral, City, University of London.

Fuchs, C. (2009). Some Reflections on Manuel Castells' Book "Communication Power". tripleC, 7(1): 94-108.

Fuchs, C. (2011). Foundations of Critical Media and Information Studies. New York: Routledge.

Fuchs, C. y Dyer-Witheford, N. (2013). Karl Marx@ Internet Studies.New Media \& Society, 15(5): 782-796.

García-Favaro, L. (2016). From Produsers to Shareaholics: Changing models of reader interaction in women's online magazines. tripleC: Communication, Capitalism \& Critique, 14(2): 346-379.

Grant, E. A. y Wilkinson, S. J. (2009). Understanding Media Convergence: The State of the Field. New York: Oxford University Press.

Gray, M. L. (2009). Out in the Country: Youth, Media, and Queer Visibility in Rural America. New York: New York University Press.

Green, J. y Jenkins, H. (2011). Spreadable media: How audiences create value and meaning in a networked economy. En V. Nightingale, The Handbook of Media Audiences (pp. 109127). Malden, MA: Wiley-Blackwell.

Gregg, M. (2009). Learning to (love) labour: Production cultures and the affective turn. Communication and Critical/Cultural Studies, 6(2): 209-214.

Haraway, D. (1991). Simians, Cyborgs and Women: The Reinvention of Nature. New York: Routledge.

Harcourt, W. (1999).Women@Internet. Creating New Cultures in Cyberspace. New York: ZedBooks.

Hardey, M. (2002). Life beyond the screen: Embodiment and identity through the Internet. The Sociological Review, 50(4): 570-585.

Hardt, M. y Negri, A. (2004). Multitude. London: Penguin.

Hay, J. y Couldry, N. (2011). Rethinking Convergence/Culture: An Introduction. Cultural Studies, 25(4-5): 473-486.

Haythornthwaite, C. y Wellman, B. (2002). (Eds) The Internet in Everyday Life. Blackwell: Oxford.

Herring, S., Johnson, D. A. y DiBenedetto, T. (1995). 'This discussion is going too far!': Male resistance to female participation on the Internet. En K. Hall y M. Bucholtz, Gender Articulated: Language and the Socially Constructed Self (pp. 67-96). New York: Routledge.

Hine, C. (2000). Virtual Ethnography. London: Sage.

Jane, E. (2014). 'Your a ugly, whorish, slut': Understanding e-bile. Feminist Media Studies, 14(4): 531-46.

Jarrett, K. (2003). Labour of Love: An Archeology of Affect as Power in Ecommerce. Journal of Sociology, 39(4): 335-51.

Jarrett, K. (2014). The relevance of "women's work": Social reproduction and immaterial labour in digital media. Television and New Media, 15(1): 14-29.

Jenkins, H. (2006). Convergence Culture: Where Old and New Media Collide. New York: New York University Press.

Jenkins, H. (2009). If it doesn't spread, it's dead (part one): Media viruses and memes. Confessions of an Aca-Fan: The Official Weblog of Henry Jenkins. Disponible http:// henryjenkins.org/blog/2009/02/if_it_doesnt_spread_its_dead_p.html 
Jenkins, H. y Deuze, M. (2008). Editorial: Convergence culture. Convergence: The International Journal into New Media Technologies, 14(1): 5-12.

Jenkins, H., Ford, S. y Green, J. (2013). Spreadable Media: Creating Value and Meaning in a Networked Culture. New York: New York University Press.

Jhally, S. y Livant, B. (1986). Watching as working: The valorization of audience consciousness. Journal of Communication, 36(3): 124-143.

Keller, J. (2015). Girls' Feminist Blogging in a Postfeminist Age. New York: Routledge.

Kendall, L. (1999). Recontextualizing 'Cyberspace': Methodological Considerations for OnLine Research. En S. Jones, Doing Internet research: critical issues and methods for examining the Net (pp. 57-75). Thousand Oaks: Sage.

Kramarae, C. y Taylor, H. J. (1993). Women and men on electronic networks: A conversation or a monologue? En H. J. Taylor, C. Kramarae y M. Ebben, Women, Information Technology, and Scholarship (pp. 52-61). Urbana, IL: Center for Advanced Study.

Lévy, P. (1997). Collective Intelligence: Mankind's Emerging World in Cyberspace. Cambridge: Perseus Books.

Livingstone, S. (2005). Critical Debates in Internet Studies: Reflections on an Emerging Field. En J. Curran y M. Gurevitch, Mass Media and Society (pp. 9-28) (5 edición). London: Sage.

McGuigan, J. (2009). Cool Capitalism. London: Pluto.

Morgan, B. (2015). Social sharing 'increases receptivity to advertising'. ResearchLive. Disponible en https:/www.research-live.com/article/news/social-sharing-increases-receptivity-to-advertising/ id/4013703

Ouellette, L. y Wilson, J. (2011). Women's work: Affective labour and convergence culture. Cultural Studies, 25(4-5): 548-565.

Paasonen, S. (2002). The woman question: Addressing women as Internet users. En M. Fernandez, F. Wilding y M. M. Wright, Domain Errors: Cyberfeminist Practice (pp. 89108). New York: Andromeda.

Pearce, C. (1997). The Interactive Book. A Guide to the Interactive Revolution. Indianapolis: Macmillan.

Plant, S. (1996). On the matrix: Cyberfeminist simulations. En R. Shields, Cultures of Internet: Virtual Spaces, Real Histories, Living Bodies (pp. 170-183). London: Sage.

Plant, S. (1997). Zeros and Ones: Digital Women and the New Technoculture. London:

Postigo, H. (2009). America online volunteers: Lessons from an early co-production community. International Journal of Cultural Studies, 12(5): 451-469.

Reid, E. (1995). Virtual worlds: Culture and imagination. En S. Jones, Cybersociety: Computer-mediated Communication and Community (pp. 164-183). London: Sage.

Rheingold, H. (1993). The Virtual Community: Homesteading on the Electronic Frontier. Reading, MA: Addison-Wesley.

Royal, C. (2005). Gendered Spaces and Digital Discourse: Framing Women's Relationship with the Internet. Tesis doctoral, The University of Texas at Austin.

Scholz, T. (2013) (Ed.) Digital Labour: The Internet as Playground and Factory. New York: Routledge.

Seiter, E. (2003). Television and the Internet. En G. Dines y J. M. Humez, Gender, Race and Class in Media (pp. 688-695). Thousand Oaks, CA: Sage.

Shade, L. (1994). Gender Issues in Computer Networking. En A. Adam, J. Emms, E. Green y J. Owen, Women, Work, Computerization: Breaking Old Boundaries, Building New Forms (pp. 91-105). Amsterdam: Elsevier. 
Smythe, D. (1977). Communications: Blindspot of western Marxism. Canadian Journal of Political and Social Theory, 1(3): 1-27.

Spender, D. (1995). Nattering on the Net. North Melbourne, Victoria: Spinifex Press.

Stone, A. R. (1995). The War of Desire and Technology at the Close of the Mechanical Age. Cambridge, MA: MIT Press.

Sundén, J. (2002). 'I'm still not sure she's a she': Textual talk and typed bodies in online interaction. En P. McIlvenny, Talking Gender and Sexuality (pp. 289-312). Amsterdam/ Philadelphia: John Benjamin.

Terranova, T. (2000). Free labour: Producing culture for the digital economy. Social Text 63, $18(2): 33-58$.

The Debrief (2016). Media pack. Bauer Media. Disponible en http://www.bauermedia.co.uk/ uploads/The_Debrief_Media_Pack_2016_TEST.pdf

Toffler, A. (1980). The third wave: The classic study of tomorrow. New York, NY: Bantam.

Turkle, S. (1995). Life on the Screen: Identity in the Age of Internet. New York: Simon and Schuster.

Van Doorn, N., Wyatt, S. y van Zoonen, L. (2008). A body of text. Feminist Media Studies, 8(4): 357-374.

Van Zoonen, L. (1992). Feminist Theory and Information Technology. Media, Culture \& Society, 14(1): 9-31.

Van Zoonen, L. (2002). Gendering the Internet: Claims, controversies and cultures. European Journal of Communication, 17(5): 5-23.

Wajcman, J. (1991). Feminism Confronts Technology. Cambridge: Polity.

Wajcman, J. (2010). Feminist theories of technology. Cambridge Journal of Economics, 34: 143-152.

Watson, N. (1997). Why we argue about virtual community: A case study of the Phish.Net fan community. En S. Jones, Virtual culture: Identity \& communication in cybersociety (pp. 102-132). London: Sage.

Wellman, B. (2011). Studying the Internet through the ages. En M. Consalvo y C. Ess, The Handbook of Internet Studies (pp. 17-23). Oxford: Oxford University Press.

Wilson, S. M. y Peterson, L. C. (2002). The anthropology of online communities. Annual Review of Anthropology, 31: 449-467.

Worthington, N. (2005). Women's work on the World Wide Web: How a new medium represents an old problem. Popular Communication: The International Journal of Media and Culture, 3(1): 43-60.

Youngs, G. (1999). Virtual voices: Real lives. En W. Harcourt, Women@Internet: Creating New Cultures in Cyberspace (pp. 55-68). London: Zed Books. 